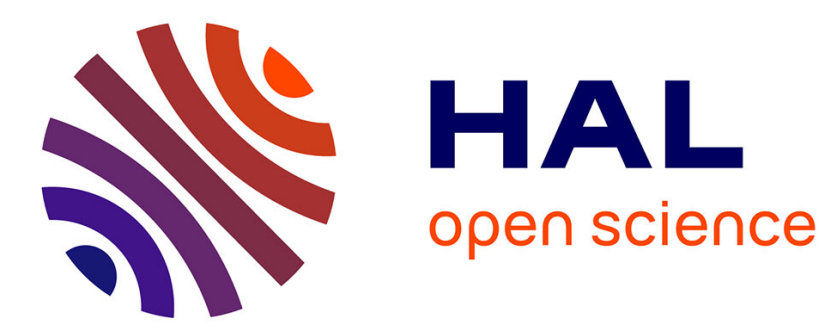

\title{
Probabilistic model identification of the bit-rock-interaction-model uncertainties in nonlinear dynamics of a drill-string
}

\author{
T.G. Ritto, Christian Soize, R. Sampaio
}

\section{- To cite this version:}

T.G. Ritto, Christian Soize, R. Sampaio. Probabilistic model identification of the bit-rock-interactionmodel uncertainties in nonlinear dynamics of a drill-string. Mechanics Research Communications, 2010, 37 (6), pp.584-589. 10.1016/j.mechrescom.2010.07.004 . hal-00684312

\section{HAL Id: hal-00684312 \\ https://hal.science/hal-00684312}

Submitted on 1 Apr 2012

HAL is a multi-disciplinary open access archive for the deposit and dissemination of scientific research documents, whether they are published or not. The documents may come from teaching and research institutions in France or abroad, or from public or private research centers.
L'archive ouverte pluridisciplinaire HAL, est destinée au dépôt et à la diffusion de documents scientifiques de niveau recherche, publiés ou non, émanant des établissements d'enseignement et de recherche français ou étrangers, des laboratoires publics ou privés. 


\title{
Probabilistic model identification of the bit-rock-interaction-model uncertainties in nonlinear dynamics of a drill-string
}

\author{
T.G. Ritto ${ }^{* a, b}$, C. Soize ${ }^{b}$, R. Sampaio ${ }^{a}$ \\ ${ }^{a}$ Department of Mechanical Engineering, PUC-Rio, Rua Marquês de São Vicente, 225, 22453-900, Rio de Janeiro, Brazil \\ ${ }^{b}$ Université Paris-Est, Laboratoire Modelisation et Simulation Multi-Echelle (MSME FRE3160 CNRS), 5 bd Descartes, 77454 \\ Marne-la-Vallée, France
}

\begin{abstract}
This paper deals with a procedure to perform the identification of the probabilistic model of uncertainties in a bit-rock interaction model for the nonlinear dynamics of a drill-string. The bit-rock interaction model is represented by a nonlinear constitutive equation, whose uncertainties are modeled using the nonparametric probabilistic approach. The identification of the parameter of this probabilistic model is carried out using the Maximum Likelihood method together with a statistical reduction in the frequency domain using the Principal Component Analysis.
\end{abstract}

Key words: Probabilistic identification, Maximum Likelihood Method, Bit-rock interaction, Stochastic dynamical system, Drill-string nonlinear dynamics

\section{Introduction}

In Ritto et al. (2009), a probabilistic model has been proposed for the bit-rock interaction model, whose uncertainties are modeled using the nonparametric probabilistic approach (Soize $(2001,2005,2010)$ ). The present paper proposes a procedure for the probabilistic identification of the bit-rock interaction model. This bit-rock interaction comes from the dynamics of a drill-string system (see, for instance, Christoforou and Yigit (1997); Khulief and Al-Naser (2005); Sampaio et al. (2007); Tucker and Wang (1999)), and it is represented by a nonlinear constitutive equation (Tucker and Wang (2003)).

There are different models in the literature for the bit-rock interaction, for example, autonomous models (Challamel (2000); Navarro-Lopez (2009); Tucker and Wang (2003)) or the distributed delay model (Germay et al. (2009)). The bit-rock interaction might lead to what is called stick-slip phenomenon, when the bit gets stuck (zero rotational speed) and then is released (slips). The present paper aims to propose a probabilistic identification procedure, thus, further analysis of bit-rock interaction models or stick-slip phenomenon will not be addressed. It should be noticed that the proposed identification procedure can be applied for different bit-rock interaction models.

With the discovery of new oil reservoirs, there is an increasing interest on the development of numerical models to support engineer decisions. In this context, uncertainties should be taken into account in the computational model in order to improve the robustness of the numerical predictions. The dynamics of a drill-string is highly affected by the bit-rock interaction, hence, this interaction is of upmost importance for the drill-string dynamics. In addition, modeling the bit-rock interaction is not trivial, and simple models are usually considered in the analysis. This is an important constraint when uncertainties are to be taken into account, since the parametric probabilistic approach limits the analysis. Therefore, in Ritto et al. (2009) it has been proposed to use the nonparametric probabilistic approach to model uncertainties in the bit-rock interaction model of a drill-string system. This probabilistic approach is able to take into account both system parameter and model uncertain-

\footnotetext{
${ }^{*}$ Corresponding author

Email addresses: thiagoritto@gmail.com (T.G. Ritto), christian.soize@univ-paris-est.fr (C. Soize), rsampaio@puc-rio.br (R. Sampaio)
} 
ties. Such a nonparametric probabilistic approach uses the random matrix theory for which the probability distributions are constructed using the Maximum Entropy Principle (Jaynes (2003)).

In the context of drill-string dynamics identification, Berzi and R. Beccu (1996) do the identification of the nonlinear dissipative spring mass model of a coupling sleeve joint, where the identification is done minimizing the distance (calculated using the Euclidean norm) between numerical and experimental responses. At the best of the authors knowledge, a probabilistic drill-string dynamics identification has not yet been investigated in the literature. Recently, Soize et al. (2008) present some strategies for probabilistic identification in uncertain computational models for dynamical systems; in particular, this paper presents the use of the maximum likelihood method (Aldrich (1997); Serfling (1980); Spall (2003)) in the case for which the output observation vector of the dynamical system is a correlated stochastic process indexed by an uncountable set. For such a case, it is proposed (Soize et al. (2008)) to apply the maximum likelihood method to the uncorrelated random variables corresponding to the coordinates of the Principal Component Analysis (PCA) of the correlated stochastic process modeling the output observation. Below, we propose to reuse such an approach to identify the probabilistic model parameter $\delta$ (which measures the uncertainty level of the bit-rock interaction nonparametric probabilistic model) in the context of nonlinear dynamics. The correlated stochastic process which models the time dependent output observation vector (which is obtained by solving the nonlinear stochastic dynamical equation of the drill-string system) is reduced using the PCA. Then, the maximum likelihood method is used to identify the uncertainties of a nonlinear constitutive equation (bit-rock interaction model). In the present analysis, we aim to validate this procedure with an experimental response that is actually generated numerically (such a procedure could then be applied using the experimental response of a real drill-string system).

\section{Deterministic model}

The deterministic model is briefly presented in this section; for a more detailed explanation of the model see Ritto et al. (2009). The excitation of the dynamical system is an imposed constant rotational speed applied at the top. The supporting force at the top (weight-on-hook) is constant, the stabilizers are modeled as linear springs, the impact forces between the column and the borehole are modeled with linear springs and the rubbing between the column and the borehole is modeled as a frictional torque. The bit-rock interaction model applied in the analysis is the one developed by Tucker and Wang (2003) and the fluid-structure interaction model used follows the model developed in Paidoussis et al. (2008). This model considers the fluid that flows downwards inside the column and then flows upwards in the annulus.

The nonlinear Timoshenko beam theory is applied to the column, in which finite strains are taken into account; the finite strains couple axial, lateral and torsional vibrations. It is assumed small rotations about the $y$ and $z$-axis and small displacements in $y$ and $z$ directions. The deterministic computational model is obtained using the finite element method and then it is reduced using a modal basis. The model is written as (Ritto et al. (2009)):

$$
\begin{aligned}
& {\left[M_{r}\right] \ddot{\mathbf{q}}(t)+\left[C_{r}\right] \dot{\mathbf{q}}(t)+\left[K_{r}\right] \mathbf{q}(t)=[\Phi]^{T}\left\{\mathbf{f}_{\mathrm{ip}}(\overline{\mathbf{u}}(t))+\mathbf{f}_{\mathrm{br}}(\overline{\mathbf{u}}(t))-\mathbf{f}_{\mathrm{NL}}(\overline{\mathbf{u}}(t), \dot{\overline{\mathbf{u}}}(t), \ddot{\mathbf{u}}(t))+\mathbf{g}(t)\right\}} \\
& \mathbf{q}(0)=\mathbf{q}_{0} \quad, \quad \dot{\mathbf{q}}(0)=\mathbf{v}_{0},
\end{aligned}
$$

where $\mathbf{q}_{0}$ and $\mathbf{v}_{0}$ are the initial conditions and $\overline{\mathbf{u}}(t)=[\Phi] \mathbf{q}(t)$ is the response of the system around the prestressed state, $\overline{\mathbf{u}}(t)=\mathbf{u}(t)-\mathbf{u}_{\mathrm{S}}$, where $\mathbf{u}_{\mathrm{S}}=[K]^{-1}\left(\mathbf{f}_{g}+\mathbf{f}_{c}+\mathbf{f}_{\mathrm{f}}\right)$. In this expression $\mathbf{f}_{g}$ is the gravity, $\mathbf{f}_{c}$ is the concentrated reaction force at the bit and $\mathbf{f}_{\mathrm{f}}$ is the fluid axial force. The reduced-order model is constructed using a modal basis selected from the normal modes of the prestressed structure. The dimension of the modal matrix $[\Phi]$ made up of these normal modes is $m \times n$, where $n$ is the number of normal modes selected to construct the reducedorder model and $m$ is the number of degrees of freedom of the finite element model. The normal modes that compose matrix $[\Phi]$ must be conveniently chosen (so that axial, lateral and torsional modes are included) in order that convergence of the response be reached.

The reduced mass, damping and stiffness matrices are written as $\left[M_{r}\right]=[\Phi]^{T}[M][\Phi],\left[C_{r}\right]=[\Phi]^{T}[C][\Phi]$ and $\left[K_{r}\right]=[\Phi]^{T}\left([K]+\left[K_{\mathrm{g}}\left(\mathbf{u}_{\mathrm{S}}\right)\right]\right)[\Phi]$. The mass $[M]$, damping $[C]$ and stiffness $[K]$ finite element matrices include the structure and fluid parts, $\left[K_{\mathrm{g}}\right]$ is the geometric stiffness matrix, $\mathbf{f}_{\mathrm{NL}}$ is the nonlinear force vector related to the nonlinear terms of the kinetic and strain energy of the structure, $\mathbf{f}_{\mathrm{ip}}$ is the force vector due to 
impact and rubbing, $\mathbf{f}_{\mathrm{br}}$ is the force vector due to the bit-rock interaction and $\mathbf{g}$ is the source force vector that appears due to the Dirichlet boundary condition (constant rotational speed about the $x$-axis at the top). The proportional damping matrix is such that $[C]=\alpha[M]+\beta\left([K]+\left[K_{\mathrm{g}}\left(\mathbf{u}_{\mathrm{S}}\right)\right]\right)$ where $\alpha$ and $\beta$ are two positive real constants.

\section{Probabilistic model}

The probabilistic model introduced by Ritto et al. (2009) to take into account uncertainties in the bit-rock interaction model is briefly summarized. The nonparametric probabilistic approach (Soize $(2000,2010)$ ) is used. It consists in modeling the operator of the bit-rock interaction constitutive equation by a random operator depending of the random state of the system. Such an approach allows both system-parameters uncertainties and modeling errors to be globally taken into account. Let the generalized forces and velocities at the bit, $f_{\text {bit }}(\dot{\mathbf{x}}(t))$ and $\dot{\mathbf{x}}(t)$, be such that $\mathfrak{f}_{\text {bit }}(\dot{\mathbf{x}}(t))=\left(f_{\text {bit }}(\dot{\mathbf{x}}(t)) \quad t_{\text {bit }}(\dot{\mathbf{x}}(t))\right)^{T}$ and $\dot{\mathbf{x}}(t)=\left(\dot{u}_{\text {bit }}(t) \omega_{\text {bit }}(t)\right)^{T}$, where $f_{\text {bit }}$ is the force at the bit (or weight-on-bit), $t_{\text {bit }}$ is the torque at the bit (or torque-on-bit), $\dot{u}_{\text {bit }}(t)$ is the axial speed of the bit (or rate of penetration) and $\omega_{\text {bit }}(t)$ is the rotational speed of the bit. The deterministic constitutive equation of the bit-rock interaction (Tucker and Wang (2003)) can be rewritten as

$$
\mathfrak{f}_{\text {bit }}(\dot{\mathbf{x}}(t))=-\left[A_{b}(\dot{\mathbf{x}}(t))\right] \dot{\mathbf{x}}(t)
$$

with

$$
\begin{array}{rlrl}
{\left[A_{b}(\dot{\mathbf{x}}(t))\right]_{11}} & =\frac{a_{1}}{a_{2} \dot{u}_{\mathrm{bit}}(t)}+\frac{1}{a_{2} Z\left(\omega_{\mathrm{bit}}(t)\right)^{2}}-\frac{a_{3} \omega_{\mathrm{bit}}(t)}{a_{2} Z\left(\omega_{\mathrm{bit}}(t)\right) \dot{u}_{\mathrm{bit}}(t)}, & {\left[A_{b}(\dot{\mathbf{x}}(t))\right]_{12}=0,} \\
{\left[A_{b}(\dot{\mathbf{x}}(t))\right]_{22}=\frac{a_{4} Z\left(\omega_{\mathrm{bit}}(t)\right)^{2} \dot{u}_{\mathrm{bit}}(t)}{\omega_{\mathrm{bit}}(t)^{2}}+\frac{a_{5} Z\left(\omega_{\mathrm{bit}}(t)\right)}{\omega_{\mathrm{bit}}(t)},} & {\left[A_{b}(\dot{\mathbf{x}}(t))\right]_{21}=0,}
\end{array}
$$

in which $a_{1}, \ldots, a_{5}$ are positive constants that depend on the bit and rock characteristics as well as on the average weight-on-bit, and where $Z$ is a regularization function. For all deterministic velocity $\dot{\mathbf{x}}(t),\left[A_{b}(\dot{\mathbf{x}}(t))\right]$ is a positive-definite matrix which is substituted, in the context of the nonparametric probabilistic modeling of uncertainties, by a random matrix $\left[\mathbf{A}_{b}(\dot{\mathbf{x}}(t))\right]$ with values in the set $\mathbb{M}_{2}^{+}(\mathbb{R})$ of all the positive-definite symmetric $(2 \times 2)$ real matrices. Thus, the constitutive equation defined by Eq. (2) becomes a random constitutive equation which can be written as

$$
\mathfrak{F}_{\text {bit }}(\dot{\mathbf{x}}(t))=-\left[\mathbf{A}_{b}(\dot{\mathbf{x}}(t))\right] \dot{\mathbf{x}}(t) .
$$

Following the construction introduced in Ritto et al. (2009); Soize (2000, 2001), for all fixed vector $\dot{\mathbf{x}}(t)$, the probability distribution of random variable $\left[\mathbf{A}_{b}(\dot{\mathbf{x}}(t))\right]$ is constructed as follows. Using the Cholesky decomposition, the mean value of $\left[A_{b}(\dot{\mathbf{x}}(t))\right]$ and random matrix $\left[\mathbf{A}_{b}(\dot{\mathbf{x}}(t))\right]$ are written as

$$
\left[A_{b}(\dot{\mathbf{x}}(t))\right]=\left[L_{b}(\dot{\mathbf{x}}(t))\right]^{T}\left[L_{b}(\dot{\mathbf{x}}(t))\right] \quad \text { and } \quad\left[\mathbf{A}_{b}(\dot{\mathbf{x}}(t))\right]=\left[L_{b}(\dot{\mathbf{x}}(t))\right]^{T}\left[\mathbf{G}_{b}\right]\left[L_{b}(\dot{\mathbf{x}}(t))\right],
$$

in which $\left[\mathbf{G}_{b}\right]$ is a random matrix whose dispersion parameter is defined by

$$
\delta=\left\{\frac{1}{2} \mathcal{E}\left\{\left\|\left[\mathbf{G}_{\mathbf{b}}\right]-[I]\right\|_{F}^{2}\right\}\right\}^{\frac{1}{2}},
$$

where $\mathcal{E}\{\cdot\}$ is the mathematical expectation and where $\|[A]\|_{F}=\left(\operatorname{trace}\left\{[A][A]^{T}\right\}\right)^{1 / 2}$ is the Frobenius norm of matrix $[A]$. The probability distribution of $\left[\mathbf{G}_{b}\right]$ (which does not depend on $t$ ) is constructed using the Maximum Entropy Principle under the constraints defined by the available information and a generator of independent realizations is deduced as explained in Soize $(2001,2005)$. The final stochastic system can be written as (Ritto et al. (2009))

$$
\begin{aligned}
& {\left[M_{r}\right] \ddot{\mathbf{Q}}(t)+\left[C_{r}\right] \dot{\mathbf{Q}}(t)+\left[K_{r}\right] \mathbf{Q}(t)=[\Phi]^{T}\left\{\mathbf{f}_{\mathrm{ip}}(\mathbf{U}(t))+\mathbf{F}_{\mathrm{br}}(\mathbf{U}(t))-\mathbf{f}_{\mathrm{NL}}(\mathbf{U}(t), \dot{\mathbf{U}}(t), \ddot{\mathbf{U}}(t))+\mathbf{g}(t)\right\},} \\
& \mathbf{U}(t)=[\Phi] \mathbf{Q}(t) \quad, \quad \mathbf{q}(0)=\mathbf{q}_{0} \quad, \quad \dot{\mathbf{q}}(0)=\mathbf{v}_{0},
\end{aligned}
$$


where $\mathbf{U}$ is the random response of the stochastic system and $\mathbf{F}_{\mathrm{br}}$ is the random force related to the uncertainty of the bit-rock interaction model (see Eq. (4)).

\section{Identification procedure}

The identification of parameter $\delta$ of the probabilistic model of the bit-rock interaction is carried out using the maximum likelihood method for the random observations. In order to implement it, a statistical reduction of the random observations is performed.

\subsection{Maximum likelihood method}

Let $\{W(t, \delta), t>0\}$ be a stochastic process of the dynamical system deduced from the random response $\{\mathbf{U}(t), t>0\}$ of the stochastic dynamical system defined by Eq. (7). For the identification of parameter $\delta$ of the probabilistic model, we use the response in the frequency domain. We then introduce the random frequency spectrum $\widehat{W}(\omega, \delta)$ as the modulus of the Fourier transform of $W(t, \delta)$ with the time window $\left[t_{i}, t_{f}\right]$, such that

$$
\widehat{W}(\omega, \delta)=\left|\int_{t_{i}}^{t_{f}} e^{-i \omega t} W(t, \delta) d t\right|,
$$

with $i=\sqrt{-1}$. The time interval $\left[t_{i}, t_{f}\right]$ only includes the random forced response of $W(t, \delta)$ (transient part induced by the initial condition is vanished at time $t_{i}$ ) and $\omega$ belongs to the frequency band of the analysis $B$. This random spectrum is calculated using the stochastic model with a frequency sampling $\left\{\omega_{1}, \ldots, \omega_{n_{\omega}}\right\}$ yielding the dependent random variables $\widehat{W}\left(\omega_{1}\right), \ldots, \widehat{W}\left(\omega_{n_{\omega}}\right)$. It is assumed that only one experimental observation is available (that is generally the case for such a complex dynamical system). The frequency-sampled experimental observation corresponding to the frequency sampling of $\widehat{W}(\omega, \delta)$ is then denoted by $\widehat{w}^{\exp }\left(\omega_{1}\right), \ldots, \widehat{w}^{\exp }\left(\omega_{n_{\omega}}\right)$. The log-likelihood function $\mathrm{L}(\delta)$ is such that (see for instance Serfling (1980); Spall (2003)),

$$
\mathrm{L}(\delta)=\log _{10} p\left(\widehat{w}^{\exp }\left(\omega_{1}\right), \ldots, \widehat{w}^{\exp }\left(\omega_{n_{\omega}}\right) ; \delta\right),
$$

in which $p\left(w_{1}, \ldots, w_{n_{\omega}} ; \delta\right)$ is the joint probability density function of the dependent random variables $\widehat{W}\left(\omega_{1}\right), \ldots$, $\widehat{W}\left(\omega_{n_{\omega}}\right)$ which is estimated with the stochastic model. Parameter $\delta$ belongs to an admissible set $C_{\delta}$ and consequently, the maximum likelihood method allows the optimal value $\delta^{\text {opt }}$ of $\delta$ to be calculated solving the following optimization problem,

$$
\delta^{\mathrm{opt}}=\arg \max _{\delta \in C_{\delta}} \mathrm{L}(\delta) .
$$

If $n_{\omega}$ is not small, that is generally the case, the numerical cost for solving this optimization problem can be prohibitive. If the random variables $\widehat{W}\left(\omega_{1}\right), \ldots, \widehat{W}\left(\omega_{n_{\omega}}\right)$ were not correlated, the following approximation $\mathrm{L}(\delta)=\sum_{k=1}^{n_{\omega}} \log _{10} p_{\widehat{W}\left(\omega_{k}\right)}\left(\widehat{w}^{\exp }\left(\omega_{k}\right) ; \delta\right)$ of the log-likelihood function could be introduced in which $p_{\widehat{W}\left(\omega_{k}\right)}\left(w_{k} ; \delta\right)$ would be the probability density function of the random variable $\widehat{W}\left(\omega_{k}\right)$. Since the dependent random variables $\widehat{W}\left(\omega_{1}\right), \ldots, \widehat{W}\left(\omega_{n_{\omega}}\right)$ are correlated, such an approximation would not be correct. We then introduced a statistical reduction allowing the maximum likelihood method to be applied to uncorrelated random variables as explained in Soize et al. (2008).

\subsection{Statistical reduction}

Let $\mathbb{W}(\delta)=\left(\widehat{W}\left(\omega_{1}, \delta\right), \ldots, \widehat{W}\left(\omega_{n_{\omega}}, \delta\right)\right)$ be the $\mathbb{R}^{n_{\omega}}$-valued random variable whose mean value is $\mathbb{M}(\delta)=$ $\mathcal{E}\{\mathbb{W}(\delta)\}$ and for which the positive $n_{\omega} \times n_{\omega}$ covariance matrix is $[\mathbb{C}(\delta)]=\mathcal{E}\left\{(\mathbb{W}(\delta)-\mathbb{M}(\delta))(\mathbb{W}(\delta)-\mathbb{M}(\delta))^{T}\right\}$. These second-order moments are usually estimated using mathematical statistics with the stochastic dynamical model. The statistical reduction is then usually obtained by performing a Principal Component Analysis (see for instance Jolliffe (1986)). We then introduce the following eigenvalue problem for the covariance matrix

$$
[\mathbb{C}(\delta)] \mathbb{X}(\delta)=\lambda(\delta) \mathbb{X}(\delta) .
$$


Let $\lambda_{1}(\delta) \geq \ldots \geq \lambda_{N_{\text {red }}}(\delta)>0$ be the $N_{\text {red }}<n_{\omega}$ largest and strictly positive eigenvalues. Let $\mathbb{X}^{1}(\delta), \ldots, \mathbb{X}^{N_{\text {red }}}(\delta)$ be the associated eigenvectors which constitute an orthonormal family for the Euclidean inner product $\langle\cdot, \cdot\rangle$. Therefore the approximation $\mathbb{W}^{N_{\mathrm{red}}}(\delta)$ of $\mathbb{W}(\delta)$ is written as

$$
\mathbb{W}^{N_{\mathrm{red}}}(\delta)=\mathbb{M}(\delta)+\sum_{k=1}^{N_{\mathrm{red}}} \sqrt{\lambda_{k}(\delta)} Y_{k}(\delta) \mathbb{X}^{k}(\delta) .
$$

The random variables $Y_{1}(\delta), \ldots, Y_{N_{\mathrm{red}}}(\delta)$ are defined by

$$
Y_{k}(\delta)=\frac{1}{\sqrt{\lambda_{k}(\delta)}}<(\mathbb{W}(\delta)-\mathbb{M}(\delta)), \mathbb{X}^{k}(\delta)>
$$

and are uncorrelated, centered, second-order, real-valued random variables, that is to say, are such that $\mathcal{E}\left\{Y_{k}(\delta)\right\}=$ 0 and $\mathcal{E}\left\{Y_{j}(\delta) Y_{k}(\delta)\right\}=\delta_{j k}$, where $\delta_{j k}$ is the Kronecker delta.The reduction is effective if $N_{\text {red }}<<n_{\omega}$, and it is chosen in order that $\left.\left\|\mathbb{W}(\delta)-\mathbb{W}^{N_{\text {red }}}(\delta)\right\| \mid \leq \sqrt{\epsilon} \| \mathbb{W}(\delta)\right]\|\|$ in which $\epsilon$ is a given accuracy, where $\|\cdot\| \|$ is such that $\|\mathbb{W} \mid\|^{2}=\mathcal{E}\left\{\|\mathbb{W}\|^{2}\right\}$ and where $\|\cdot\|$ is the Euclidean norm. Therefore, $\left\|\mathbb{W}(\delta)-\mathbb{W}^{N_{\text {red }}}(\delta)\right\| \|^{2}=$ $\operatorname{tr}([\mathbb{C}(\delta)])-\sum_{k=1}^{N_{\text {red }}} \lambda_{k}(\delta)$, where $\operatorname{tr}(\cdot)$ is the trace of a matrix. It can then be deduced that $N_{\text {red }}$ has to be chosen such that

$$
N_{\text {red }}=\arg \left\{\max _{N \in\left\{1,2, . ., n_{\omega}\right\}}\left(1-\frac{\sum_{k=1}^{N} \lambda_{k}(\delta)}{\operatorname{tr}([\mathbb{C}(\delta)])}\right) \leq \epsilon\right\},
$$

Let $\mathbb{W}^{\exp }=\left(\widehat{w}^{\exp }\left(\omega_{1}\right), \ldots, \widehat{w}^{\exp } \omega_{n_{\omega}}\right)$. The experimental observations $y_{1}^{\exp }(\delta), \ldots, y_{N_{\text {red }}}^{\exp }(\delta)$ corresponding to the random variables $Y_{1}(\delta), \ldots, Y_{N_{\text {red }}}(\delta)$ are then obtained using the projection defined by Eq. (13), that is to say, they are written as

$$
y_{k}^{\exp }(\delta)=\frac{1}{\sqrt{\lambda_{k}(\delta)}}<\left(\mathbb{W}^{\exp }-\mathbb{M}(\delta)\right), \mathbb{X}^{k}(\delta)>
$$

Let $p_{Y_{1}, \ldots, Y_{N_{\mathrm{red}}}}\left(y_{1}, \ldots, y_{N_{\mathrm{red}}} ; \delta\right)$ be the joint probability density function of the random variables $Y_{1}, \ldots, Y_{N_{\mathrm{red}}}$. The log-likelihood function is such that $\mathrm{L}^{\mathrm{red}}(\delta)=\log _{10} p_{Y_{1}, \ldots, Y_{N_{\mathrm{red}}}}\left(y_{1}^{\exp }(\delta), \ldots, y_{N_{\mathrm{red}}}^{\exp }(\delta) ; \delta\right)$. Since random variables $Y_{1}, \ldots, Y_{N_{\text {red }}}$ are uncorrelated (but dependent), it is now reasonable to introduce the following simplification for the log-likelihood function which is then rewritten as

$$
\mathrm{L}^{\mathrm{red}}(\delta)=\sum_{k=1}^{N_{\mathrm{red}}} \log _{10} p_{Y_{k}}\left(y_{k}^{\exp }(\delta) ; \delta\right),
$$

in which $p_{Y_{k}}\left(y_{k} ; \delta\right)$ is the probability density function of random variable $Y_{k}(\delta)$ which is estimated with the statistical reduction model. The optimization problem is then rewritten as

$$
\delta^{\mathrm{opt}}=\arg \max _{\delta \in C_{\delta}} \mathrm{L}^{\mathrm{red}}(\delta) .
$$

\section{Numerical results}

The 1600 meters length drill-string defined in Ritto et al. (2009) with data given in Appendix A is considered. For the construction of the reduced dynamical model, 158 lateral modes, 4 torsional modes, 3 axial modes and two rigid body modes (axial and torsional) of the structure are used. As initial conditions, the column is moving axially with velocity $4.2 \times 10^{-3} \mathrm{~m} / \mathrm{s}$, rotating around the $x$-axis with $5.24 \mathrm{rd} / \mathrm{s}$ and deflected laterally. The system is excited by a constant rotational speed at the top of $5.24 \mathrm{rd} / \mathrm{s}$. For the time integration procedure, the implicit Newmark integration scheme has been implemented with a predictor and a fix point algorithm in order that the system be in equilibrium at each time step which is $\Delta t=5 \times 10^{-5}$. All the numerical results presented below correspond to the forced response for which the transient part of the response induced by the initial conditions has vanished. The time window is $\left[t_{i}, t_{f}\right]=[150,250] \mathrm{s}$. The frequency band of analysis is $B=[0,1.5] \mathrm{Hz}$. The frequency and the time samplings correspond to $n_{\omega}=250$ and $n_{t}=10000$. To simplify the numerical analysis 
and to speed-up the computational time, only stable behaviors are considered (no stick phase). Nevertheless, the proposed probabilistic identification procedure is applied also for responses with stick-slip oscillations.

Figures 1 and 2 show the forced dynamical response of the system without uncertainties (deterministic). Figure 1(a) shows the axial displacement of the bit and Fig. 1(b) shows the axial speed at $x=700 \mathrm{~m}$. It can be noticed that the drill-string is moving forward. Figure 2(a) shows the rotation of the bit versus the rotational speed of the bit and Fig. 2(b) shows the frequency spectrum of the rotational speed of the bit. This frequency spectrum is going to be used in the stochastic analysis, as explained latter.
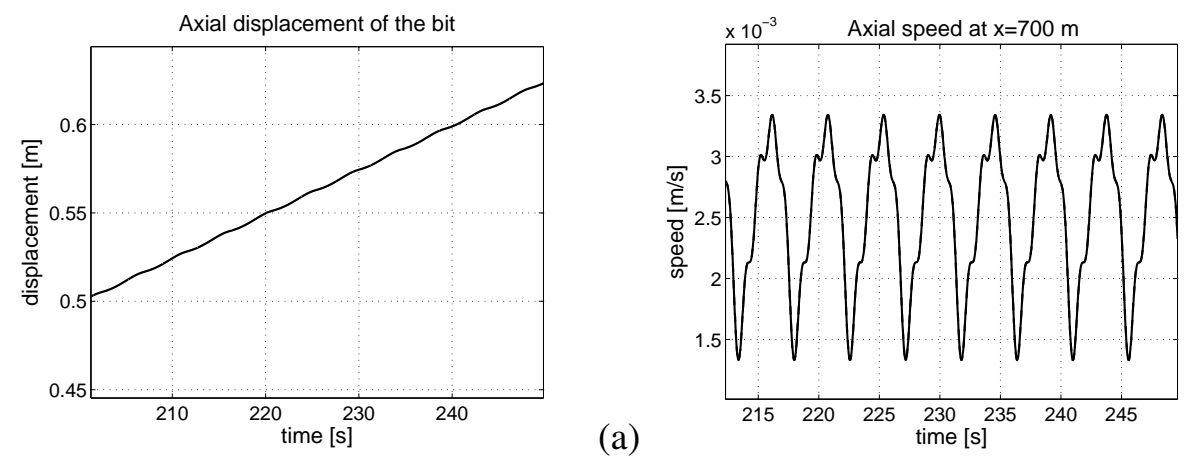

Fig. 1: (a) axial displacement of the bit and (b) axial speed at $x=700 \mathrm{~m}$.
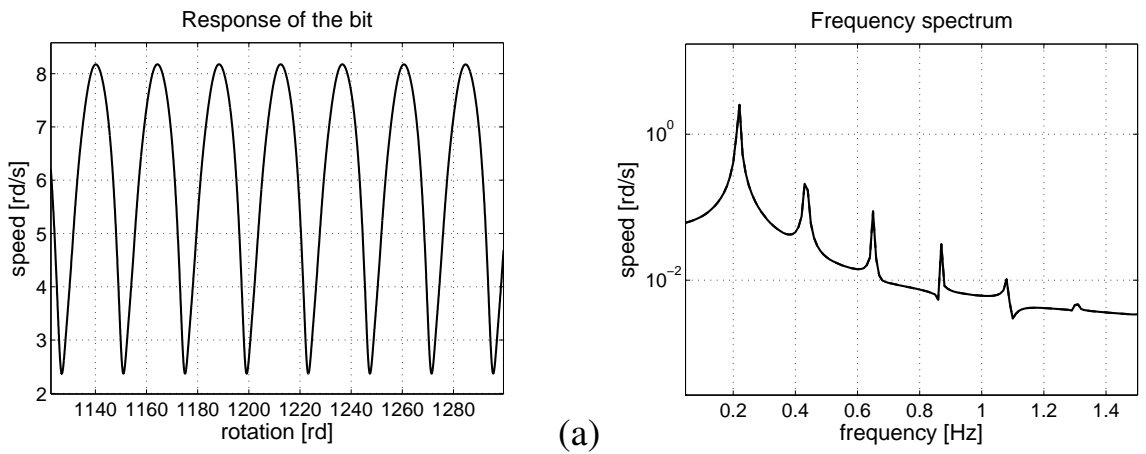

(b)

Fig. 2: (a) rotation of the bit versus rotational speed of the bit and (b) frequency spectrum of the rotational speed of the bit.

There are some measurement equipment that might measure the dynamics of a real drill-string system, while drilling. Among the measurements, one that is important for the analysis is the rotational speed of the bit. Therefore, this dynamical response is used as observation for the identification procedure and then the frequency spectrum observed $\widehat{W}(\omega, \delta)$ is the frequency spectrum $\widehat{\mathcal{W}}_{\text {bit }}(\omega, \delta)$ of the random rotational speed of the bit. The corresponding experiments $\widehat{\mathcal{W}}_{\text {bit }}^{\exp }(\omega, \delta)$ has been generated numerically for the present analysis. Figure 3(a) shows the convergence of the stochastic analysis, where $\operatorname{conv}\left(n_{s}\right)=\frac{1}{n_{s}} \sum_{i=1}^{n_{s}} \int_{t_{i}}^{t_{f}}\left\|\mathbf{U}\left(t, \theta_{i}\right)\right\|^{2} d t$ in which $n_{s}$ is the number of Monte Carlo simulations.

The trial method is used to solve the optimization problem defined by Eq. (17). The stochastic nonlinear dynamical model is solved for $\delta$ in $\{0.01,0.02,0.03,0.04,0.05,0.06,0.07,0.08,0.09,0.10\}$. The value of $\epsilon$ is set $\epsilon=1 \times 10^{-4}$ yielding $N_{\text {red }}=40$ (see Eq. (14)). Figure 3(b) shows how the log-likelihood L ${ }^{\text {red }}$ (see Eq. (16)) varies with the dispersion parameter $\delta$. We conclude that the most likely value for the dispersion parameter $\delta^{\text {opt }}$ is 0.06 .

Figures 4 and 5 show the response of the stochastic system for the identified value 0.06 of $\delta$. Figure 4(a) shows random realizations of the rotational speed of the bit $\mathcal{W}_{\text {bit }}$ and Fig. 4(b) shows the coefficient of variation $\delta_{W}(t)=\sigma_{W}(t) / \mu_{W}(t)$ of the random rotational speed of the bit at each time $t$ in which $\sigma_{W}(t)$ is the standard deviation and $\mu_{W}(t)$ is the mean value of $\mathcal{W}_{\mathrm{bit}}(t)$ at each instant $t$. Although $\delta$ is small (0.06), the coefficient of variation of the response is significant $\left(\delta_{W} \sim 0.4\right)$. Figure 5 shows the statistical envelope of the frequency 

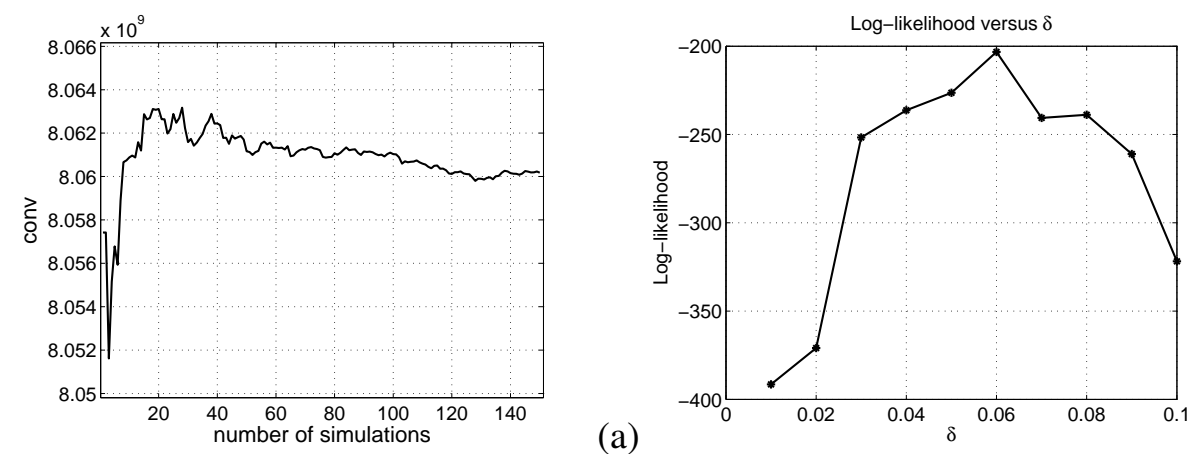

(b)

Fig. 3: (a) convergence function and (b) log-likelihood function.

spectrum $\widehat{W}_{\text {bit }}$ of the random rotational speed of the bit together with the response of the deterministic system and the mean response of the stochastic system.
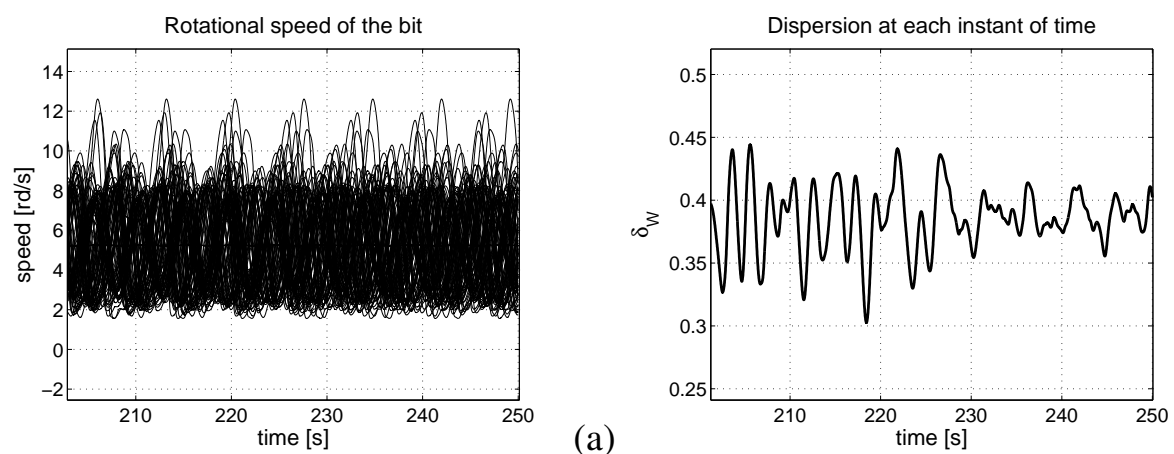

(b)

Fig. 4: (a) random realizations of the rotational speed of the bit for $\delta=0.06$ and (b) coefficient of variation of $\mathcal{W}_{\text {bit }}$ at each instant for $\delta=0.06$.

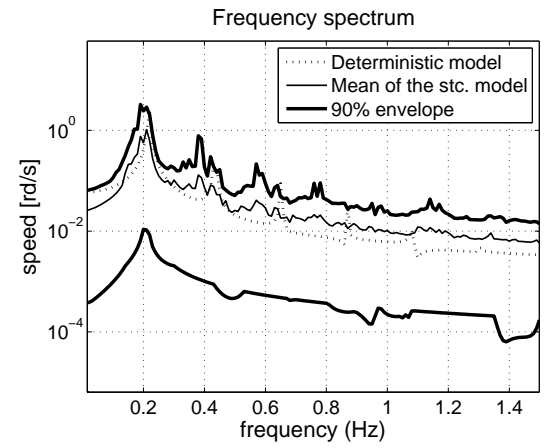

Fig. 5: $90 \%$ statistical envelope of $\widehat{\mathcal{W}}_{\text {bit }}$ for $\delta=0.06$ together with the deterministic response and the mean of the stochastic response.

\section{Concluding Remarks}

A procedure has been proposed to identify the probabilistic model of a bit-rock interaction model in the context of drill-string dynamics. The uncertainties related to the nonlinear constitutive equation of the interaction model are modeled using the nonparametric probabilistic approach. The maximum likelihood method has been employed together with a statistical reduction. The proposed procedure has been validated using an experimental response generated numerically. Such a procedure can then by applied to an experimental response of a real drill-string system. 


\section{Acknowledgements}

The authors acknowledge the financial support of the Brazilian agencies CNPQ, CAPES and FAPERJ, and the French agency COFECUB (project CAPES-COFECUB 476/04).

\section{A. Data used in the simulation}

$L_{\mathrm{dp}}=1400 \mathrm{~m}$ (length of the drill pipe)
$D_{\text {odp }}=0.127 \mathrm{~m}$ (outside diameter, drill pipe)
$D_{\text {idp }}=0.095 \mathrm{~m}$ (inside diameter, drill pipe)
$D_{\mathrm{ch}}=0.3 \mathrm{~m}$ (diameter of the borehole)
$k_{\mathrm{stab}}=17.5 \mathrm{MN} / \mathrm{m}$ (stabilizer stiffness)
$\rho=7850 \mathrm{~kg} / \mathrm{m}^{3}$ (density)
$k_{s}=6 / 7$ (shearing correcting factor)
$\mu_{\text {ip }}=0.0005$ (frictional coefficient)
$\rho_{\mathrm{f}}=1200 \mathrm{~kg} / \mathrm{m}^{3}$ (density of the fluid)
$k=0$ (fluid viscous damping coefficient)
$a_{1}=3.429 \times 10^{-3} \mathrm{~m} / \mathrm{s}$ (cte of the bit-rock interac.)
$a_{3}=1.374 \times 10^{-4} \mathrm{~m} / \mathrm{rd}$
$a_{5}=1.475 \times 10^{3} \mathrm{~N} . \mathrm{m}$

$L_{\mathrm{dc}}=200 \mathrm{~m}$ (length of the drill collar)

$D_{\text {odc }}=0.2286 \mathrm{~m}$ (outside diameter, drill collar)

$D_{i \mathrm{dc}}=0.0762 \mathrm{~m}$ (inside diameter, drill collar)

$x_{\text {stab }}=1400 \mathrm{~m}$ (location of the stabilizer)

$E=210 \mathrm{GPa}$ (elasticity modulus)

$v=0.29$ (poisson coefficient)

$k_{\text {ip }}=1 \times 10^{8} \mathrm{~N} / \mathrm{m}$ (stiffness of the impacts)

$U_{i 0}=4 \mathrm{~m} / \mathrm{s}$ (inlet flow velocity)

$C_{\mathrm{f}}=0.0125$ (fluid viscous damping coefficient)

$g=9.81 \mathrm{~m} / \mathrm{s}^{2}$ (gravity acceleration)

$a_{2}=5.672 \times 10^{-8} \mathrm{~m} /(\mathrm{N} . \mathrm{s})$

$a_{4}=9.537 \times 10^{6} \mathrm{~N} \cdot \mathrm{rd}$

$e=2 \mathrm{rd} / \mathrm{s}$ (regularization parameter).

The damping matrix is constructed using the relationship $[C]=\alpha\left([M]+\left[M_{\mathrm{f}}\right]\right)+\beta\left([K]+\left[K_{\mathrm{f}}\right]+\left[K_{\mathrm{g}}\left(\mathbf{u}_{\mathrm{S}}\right)\right]\right)$ with $\alpha=0.01$ and $\beta=0.0003$.

\section{References}

Aldrich, J., 1997. R.A. Fisher and the making of maximum likelihood 1912-1922. Statistical Science 12 (3), $162-176$.

Berzi, P., R. Beccu, B. L., 1996. Identification of a percussive drill rod joint from its response to stress wave loading. International Journal of Impact Engineering 18 (3), 281-290.

Challamel, N., 2000. Rock destruction effect on the stability of a drilling structure. Journal of Sound and Vibration 233 (2), $235-254$.

Christoforou, A. P., Yigit, A. S., 1997. Dynamic modeling of rotating drillstrings with borehole interactions. Journal of Sound and Vibration 206 (2), 243-260.

Germay, C., Denoel, V., Detounay, E., 2009. Multiple mode analysis of the self-excited vibrations of rotary drilling systems. Journal of Sound and Vibration 325 (3), 362-381.

Jaynes, E., 2003. Probability Theory: The Logic of Science. Vol. 1. Cambridge University Press, Cambridge, UK.

Jolliffe, I. T., 1986. Principal Component Analysis. Springer-Verlag, New York, M.A. USA.

Khulief, Y., Al-Naser, H., 2005. Finite element dynamic analysis of drillstrings. Finite Elements in Analysis and Design 41, 1270-1288.

Navarro-Lopez, E. M., 2009. An alternative characterization of bit sticking phenomena in a multiple-degree-of-freedom controlled drillstring. Nonlinear Analysis: Real World Applications 10, 3162-3174.

Paidoussis, M. P., Luu, T. P., Prabhakar, S., 2008. Dynamics of a long tubular cantilever conveying fluid downwards, which then flows upwards around the cantilever as a confined annular flow. Journal of Fluids and Structures 24, 111-128.

Ritto, T. G., Soize, C., Sampaio, R., 2009. Nonlinear dynamics of a drill-string with uncertain model of the bit-rock interaction. International Journal of Non-Linear Mechanics 44 (8), 865-876.

Sampaio, R., Piovan, M. T., Lozano, G. V., 2007. Coupled axial/torsional vibrations of drilling-strings by mean of nonlinear model. Mechanics Research Comunications 34 (5-6), 497-502.

Serfling, R. J., 1980. Approximation Theorems of Mathematical Statistics. John Wiley and Sons, USA.

Soize, C., 2000. A nonparametric model of random uncertities for reduced matrix models in structural dynamics. Probabilistic Engineering Mechanics 15, 277-294.

Soize, C., 2001. Maximum entropy approach for modeling random uncertainties in transient elastodynamics. Journal of the Acoustical Society of America 109(5), 1979-1996.

Soize, C., 2005. Random matrix theory for modeling uncertainties in computational mechanics. Computer Methods in Applied Mechanics and Engineering 194 (12-16), 1333-1366.

Soize, C., 2010. Generalized probabilistic approach of uncertainties in computational dynamics using random matrices and polynomial chaos decompositions. International Journal for Numerical Methods in Engineering, Accepted: 12 June 2009, on line DOI $10.1002 / \mathrm{nme} .2712$.

Soize, C., Capiez-Lernout, E., Durand, J. F., Fernandez, C., Gagliardini, L., 2008. Probabilistic model identification of uncertainties in computational models for dynamical systems and experimental validation. Computer Methods in Applied Mechanics and Engineering 198, 150-163.

Spall, J. C., 2003. Introduction to Stochastic Search and Optimization. John Wiley and Sons, Hoboken, NJ, US.

Tucker, R. W., Wang, C., 1999. An integrated model for drill-string dynamics. Journal of Sound and Vibration 224 (1), $123-165$.

Tucker, R. W., Wang, C., 2003. Torsional vibration control and Cosserat dynamics of a drill-rig assembly. Meccanica 38 (1), $143-159$. 